\title{
Combination of Ad-sTRAIL with the chemotherapeutic drug cisplatin synergistically enhances their pro-apoptotic ability in human breast cancer cells
}

\author{
XIANGPING LIU ${ }^{1 *}$, JING WANG $^{2 *}$, HAIBO WANG $^{2}$, SHIHAI LIU ${ }^{1}$, \\ YE LIANG ${ }^{1}$, ZHIDONG LV $^{2}$, QUAN ZHOU ${ }^{1}$ and WEILI DING ${ }^{2}$ \\ ${ }^{1}$ Central Laboratory of Molecular Biology and ${ }^{2}$ Center of Diagnosis and Treatment of Breast Disease, \\ The Affiliated Hospital of the Medical College, Qingdao University, Qingdao 266003, P.R. China
}

Received May 27, 2013; Accepted July 17, 2013

DOI: 10.3892/or.2013.2653

\begin{abstract}
Tumor necrosis factor-related apoptosis-inducing ligand/Apo2 ligand (TRAIL or Apo2L) is a member of the tumor necrosis factor superfamily that induces apoptosis in various cancer cell types but not in most normal cells. However, it is clear that not all cancer cells are sensitive to the killing effects of TRAIL, including breast cancer. Previous studies have demonstrated that chemotherapeutic drugs sensitize tumor cells to apoptosis induced by TRAIL in several types of malignancies. In the present study, we studied the effects of TRAIL combined with cisplatin on two breast cancer cell lines MDA-MB-468 and HCC-1937 in vitro. MTT assay, crystal violet staining assay, DAPI staining assay and flow cytometric analysis were undertaken to evaluate the enhancement of breast cancer cell death using Ad-sTRAIL and/or cisplatin. The levels of apoptotic molecules in signal transduction pathways were analyzed by real-time RT-PCR and western blotting. We found that co-treatment with Ad-sTRAIL and cisplatin exhibited stronger cytotoxicity and induced more significant apoptosis in breast cancer cells compared with Ad-sTRAIL or cisplatin alone. Pretreatment with cisplatin significantly enhanced the expression of DR5. Moreover, the induction of apoptosis by TRAIL plus cisplatin was accompanied by the downregulation of cFLIP and BCL2L1, and simultaneously robust enzymatic activation of caspase- 8 , culminating in decreased cancer cell survival. The present study revealed that TRAIL conjugated with cisplatin exhibited a markedly increased cytotoxic and apoptosis-inducing effect on breast cancer cells.
\end{abstract}

Correspondence to: Professor Haibo Wang, Center of Diagnosis and Treatment of Breast Disease, The Affiliated Hospital of the Medical College, Qingdao University, Qingdao 266003, P.R. China E-mail: hbwang66@126.com

${ }^{*}$ Contributed equally

Key words: TRAIL, cisplatin, cFLIP, apoptosis, breast cancer

\section{Introduction}

Breast cancer continues to be a major health threat to women worldwide despite the recent advances in diagnosis and treatment. It was estimated that 1.38 million new breast cancer cases were diagnosed in 2008 (23\% of all cancers) (1), and more than 400,000 people die from breast cancer each year in the world (2). Hence, it is essential to develop new anticancer drugs and novel regimens that are capable of killing cancer cells, particularly drug-resistant cells.

TRAIL preferentially exerts its obvious suppression of growth in a variety of cancer cells without influencing normal tissues and cells (3) as compared to tumor necrosis factor (TNF) and FasL which lead to severe liver damage and lethal inflammatory reactions $(4,5)$. Nevertheless, recent studies suggest that TRAIL resistance exists in many tumor cell lines (6), particularly certain highly malignant tumor cells $(7,8)$. Worse still, some cancer cells initially sensitive to TRAIL may develop resistance to TRAIL after repeated use (9). Thus, it is indicated that TRAIL alone may be ineffective for breast cancer therapy.

Fortunately, many studies have shown that chemotherapeutic drugs can lead to enhanced apoptosis induction in TRAIL-resistant tumor cells $(10,11)$. Cisplatin is a widely used conventional chemotherapeutic drug used for breast cancer treatment. By forming DNA adducts, cisplatin leads to DNA damage through p53-dependent and p53-independent mechanisms $(12,13)$. Yet, it is known to cause severe side-effects, being toxic to normal cells and organs at the concentrations essential for effective treatment of malignancies $(14,15)$.

With the development of molecular biology and research into the pathogenesis of various types of tumors, gene therapy has become a potential method for the biotherapy of tumors (16). Gene therapy applies a variety of delivery vehicles to transfer therapeutic genes to tumor cells. Adenovirus is now one of the most prospective delivery vectors for gene therapy due to advantages, such as low pathogenicity, lack of immunogenicity, infection of non-dividing cells and steady integration into the tumor cell genome at a targeted site (17). To date, adenovirus as an excellent vector has been used in many clinical trials for treating certain types of malignancies 
$(18,19)$. Moreover, adenovirus possesses a broad host range; breast cancer cells included (20).

In the present research, two breast cancer cell lines, MDA-MB-468 and HCC-1937, were treated with Ad-sTRAIL and cisplatin to investigate their antitumor efficacy in vitro and explore the molecular mechanism. The results showed that cisplatin sensitized breast cell lines to TRAIL-induced apoptosis probably by upregulating the level of DR5, downregulating the expression of cFLIP and BCL2L1 and inducing activation of caspase-8. Therefore, Ad-sTRAIL in combination with the conventional chemotherapeutic agent cisplatin has potential for clinical cancer therapy.

\section{Materials and methods}

Cell lines and reageats. Human breast cancer cell lines MDA-MB-468 and HCC-1937 were purchased from the Type Culture Collection of the Chinese Academy of Science (Shanghai, China). MDA-MB-468 cells were maintained in L-15 medium (Gibco), and HCC-1937 cells were maintained in RPMI-1640 medium (HyClone), both with $10 \%$ fetal bovine serum (FBS) (HyClone) as recommended by the Type Culture Collection. Cells were incubated under $5 \% \mathrm{CO}_{2}$ and at $37^{\circ} \mathrm{C}$. The cells were always detached using $0.25 \%$ trypsin (Amresco) and $0.02 \%$ ethylenediaminetetraacetic acid (EDTA). Cisplatin (10 mg) was purchased from Qilu Pharmaceutical Co., Ltd. (Jinan, China). The recombinant Ad-sTRAIL (the virus titer was $2.18 \times 10^{9} \mathrm{IFU} / \mathrm{ml}$ ) was constructed by the Central Laboratory, The Affiliated Hospital of the Medical College, Qingdao University. DAPI dye and crystal violet dye were obtained from Beyotime Institute of Biotechnology. RNAiso reagent (TRIzol reagent), the SuperScript ${ }^{\mathrm{TM}}$ II reverse transcriptase kit and PCR-DRR081A (used for fluorogenic quantitative RT-PCR) were obtained from Takara Biotechnology Co., Ltd. Annexin V-PE (R-phycoerythrin) and propidium iodide (PI) for flow cytometry were purchased from Invitrogen Corp. Primary antibodies against DR5 and caspase- 8 were purchased from Cell Signaling Technology. Anti-glyceraldehyde-3-phosphate dehydrogenase (GAPDH) rabbit polyclonal antibody was obtained from Abcam. Unless mentioned, all other reagents were obtained from Sigma.

MTT assay. The effect of Ad-sTRAIL and cisplatin on MDA-MB-468 and HCC-1937 cell viability was measured using the 3-(4,5-dimethylthiazol-2-yl)-2,5-diphenyltetrazolium bromide (MTT) assay. Cells $\left(4 \times 10^{4}\right)$ in $100 \mu$ l medium were seeded in each well of a 96-well plate and were left for $24 \mathrm{~h}$ to adhere. The cells were then treated with different concentrations of cisplatin for $48 \mathrm{~h}$. Thereafter, $20 \mu \mathrm{l}$ of MTT $(5 \mathrm{mg} / \mathrm{ml})$ was added into each well and incubated under $5 \% \mathrm{CO}_{2}$ and at $37^{\circ} \mathrm{C}$ for another $4 \mathrm{~h}$. After removal of the culture medium, the cells were lysed in $150 \mu \mathrm{l}$ of dimethylsulfoxide (DMSO). The plate was shaken until the crystals were dissolved. Afterwards, the absorption at $490 \mathrm{~nm}\left(\mathrm{OD}_{490}\right)$ was measured using a microplate reader (Bio-Rad Laboratories, Hercules, CA, USA). In this way, the half maximal inhibitory concentration $\left(\mathrm{IC}_{50}\right)$ of cisplatin was determined. The cells were then treated with cisplatin $(5 \mu \mathrm{g} / \mathrm{ml})$ and Ad-sTRAIL (multiplicity of infection MOI $=10$ ), alone or combined for $48 \mathrm{~h}$. The inhibitory rates of the different groups were calculated using the formula: Cell inhibitory rate $(\%)=(1-\mathrm{OD}$ of the experimental group/OD of the control group) $\mathrm{x} 100 \%$.

Crystal violet staining assay. The breast cancer cells were seeded in each well of a 24-well plate with a density of $2 \times 10^{5} /$ well at subconfluent levels. The cells were treated with various concentrations of cisplatin and were infected with Ad-sTRAIL at various MOIs, alone or combined. After 7 day of incubation at $37^{\circ} \mathrm{C}$ and $5 \% \mathrm{CO}_{2}$, the medium was removed and cells were exposed to $2 \%$ crystal violet in $20 \%$ methanol for $15 \mathrm{~min}$. The plates were then washed with deionized water until the dye was washed off and finally documented as photographs.

DAPI staining assay. The breast cancer cells were cultured in each well of a 24 -well plate with a density of $3 \times 10^{5} /$ well with $1 \mathrm{ml}$ medium. The cells were then treated with cisplatin (5 $\mu \mathrm{g} / \mathrm{ml})$ and Ad-sTRAIL (MOI=10), alone or combined. After incubation for $48 \mathrm{~h}$, cells were collected and centrifuged at 3,000 rpm for $5 \mathrm{~min}$ at room temperature, washed twice in phosphate-buffered saline (PBS), fixed with $4 \%$ formaldehyde at room temperature for $30 \mathrm{~min}$ and washed with PBS twice again after fixation. Afterwards, $200 \mu \mathrm{l}$ of 4,6-diamidino2-phenylindole (DAPI; $2 \mu \mathrm{l} / \mathrm{ml}$ ) was added into each well for incubation for $30 \mathrm{~min}$ at room temperature avoiding light exposure. After discarding the DAPI dye, cells were washed with PBS twice to remove the redundant fluorescent dye. The preparation was completely resuspended in the antifading mounting medium. The preparation was then transferred to glass slides for observation under a fluorescence microscope (DM2500; Leica Microsystems, Wetzlar, Germany) and images were captured and analyzed.

Flow cytometric analysis. Cells were seeded in each well of 6 -well plates at a density of $5 \times 10^{6}$ and treated with Ad-sTRAIL and/or cisplatin for $48 \mathrm{~h}$. Both adherent and suspended cells were collected, washed in PBS and then suspended in Annexin-binding buffer. Afterwards, cells were stained with Annexin V-PE or PI to distinguish apoptotic and dead cells. All steps were conducted in accordance with the manufacturer's instructions. Finally, the stained cells were analyzed by flow cytometry.

Real-time fluorogenic quantitative RT-PCR. Total mRNA was extracted from the cells of the 4 groups using TRIzol reagent and then quantitated using an ultraviolet spectrophotometer (Beckman Coulter) by measuring $\mathrm{A}_{260}$ and $\mathrm{A}_{280}$. Then $0.5 \mathrm{mg}$ mRNA of each sample was reversely transcribed into cDNA using the PrimeScript reverse transcriptase kit. To assess the levels of mRNA of the target gene, we used realtime fluorescence quantitative PCR analysis based on the SYBR-Green method in $\mathrm{A}_{480}$ real-time thermal cycler (Roche). The PCR reactions in duplicate were subjected to an initial denaturation at $95^{\circ} \mathrm{C}$ for $10 \mathrm{sec}$, followed by 40 cycles of denaturation at $95^{\circ} \mathrm{C}$ for $5 \mathrm{sec}$, annealing and extension at $60^{\circ} \mathrm{C}$ for $45 \mathrm{sec}$. The value of the threshold cycle $(\mathrm{Ct})$ for each reaction was recorded. The levels of target $\mathrm{mRNA}$ transcripts relative to GAPDH were expressed as the $\Delta \mathrm{Ct}\left(\mathrm{Ct}_{\text {Target }}-\mathrm{Ct}_{\mathrm{GAPDH}}\right)$ and further quantified using the $2^{-\Delta \Delta \mathrm{CT}}$ method, where $\Delta \Delta \mathrm{Ct}=$ $\left(\mathrm{Ct}_{\text {Target }}-\mathrm{Ct}_{\mathrm{GAPDH}}\right)_{\text {experimental group }}-\left(\mathrm{Ct}_{\text {Target }}-\mathrm{Ct}_{\mathrm{GAPDH}}\right)_{\text {control group }}$ (21). RT-PCR was performed with primer pairs for TRAIL: 
forward (5'-AGTGAGAGAAAGAGGTCCTCAG-3') and reverse (5'-CCAGAGCCTTTTCATTCTTGGA-3'); for cFLIP: forward (5'-AGAGTGAGGCGATTTGACCTG-3') and reverse (5'-GTCCGAAACAAGGTGAGGGTT-3'); for BCL2L1: forward (5'-GACTGAATCGGAGATGGAGACC-3') and reverse (5'-GCAGTTCAAACTCGTCGCCT-3'); for BCL2: forward (5'-ATGTGTGTGGAGAGCGTCAACC-3') and reverse (5'-TGAGCAGAGTCTTCAGAGACAGCC-3'); and for GAPDH as a control: forward (5'-TCATGGGTGTGAACCATGAGAA-3') and reverse (5'-GGCATGGACTGTGGTCATGAG-3').

Western blot analysis. Cell samples were lysed in ice-cold lysis buffer (Beyotime Institute of Biotechnology, Haimen, China) with $1 \%$ PMSF (phenylmethylsulfonyl fluoride) for half an hour, then centrifuged at $10,000 \mathrm{x} \mathrm{g}$ for $20 \mathrm{~min}$ at $4^{\circ} \mathrm{C}$, and the protein concentration of the resulting supernatant was determined using the BCA (bicinchoninic acid) protein assay kit (Beyotime Institute of Biotechnology). Proteins $(50 \mu \mathrm{g})$ were separated by $12 \%$ SDS-PAGE electrophoresis and subsequently transferred to PVDF membranes. Membranes were blocked with 5\% non-fat dry milk in TBS/Tween-20 $(0.05 \%$, $\mathrm{v} / \mathrm{v})$ for $2 \mathrm{~h}$ at room temperature and incubated overnight at $4^{\circ} \mathrm{C}$ with the primary antibodies directed against DR5, caspase- 8 and GAPDH. The blots were washed and incubated with the horseradish peroxidase-conjugated secondary antibody (DakoCytomation) and developed with a chemiluminescent substrate, ECL Plus. An autoradiograph was obtained, and protein levels were measured using a Fluor-S scanner and Quantity One software for analysis (Bio-Rad Laboratories).

Statistical analysis. All experiments were conducted at least three times, and experimental data are expressed by the means \pm SD. P-values were determined by the Student's t-test. $\mathrm{P}<0.05$ was considered to indicate a statistically significant result. $\mathrm{IC}_{50}$ values were calculated using the GraphPad Prism version 5.00 for Windows (GraphPad software, San Diego, CA, USA).

\section{Results}

Combination of Ad-sTRAIL and cisplatin promotes the apoptosis of breast cancer cells. The MDA-MB-468 and HCC-1937 cells were treated with different concentrations of cisplatin for $48 \mathrm{~h}$, and thereafter the inhibition rate was determined. Fig. 1 shows that cisplatin triggered a dose-dependent inhibition of cancer cell proliferation. Yet, when the concentration was $>30 \mu \mathrm{g} / \mathrm{ml}$, the inhibition rate increased slowly, entering into a plateau period. The $50 \%$ inhibitory concentration $\left(\mathrm{IC}_{50}\right)$ of the MDA-MB-468 cells was $15.32 \mu \mathrm{g} / \mathrm{ml}$, and that of HCC-1937 at $48 \mathrm{~h}$ was $11.91 \mu \mathrm{g} / \mathrm{ml}$. Examination of the cisplatin effect on TRAIL-induced apoptosis showed that cisplatin exhibited a synergistic effect on TRAIL-induced apoptosis in the MDA-MB-468 and HCC-1937 cell lines (Fig. 2). The flow cytometric analysis showed that the apoptotic proportion of MDA-MB-468 and HCC-1937 cells treated with Ad-sTRAIL combined with cisplatin was significantly higher (34.32 and $42.16 \%$, respectively) than the apoptotic proportion of cells treated with either agent alone (Fig. 3). The following crystal violet assay further confirmed the above observations. The images indicate that the treatment of cisplatin or Ad-sTRAIL

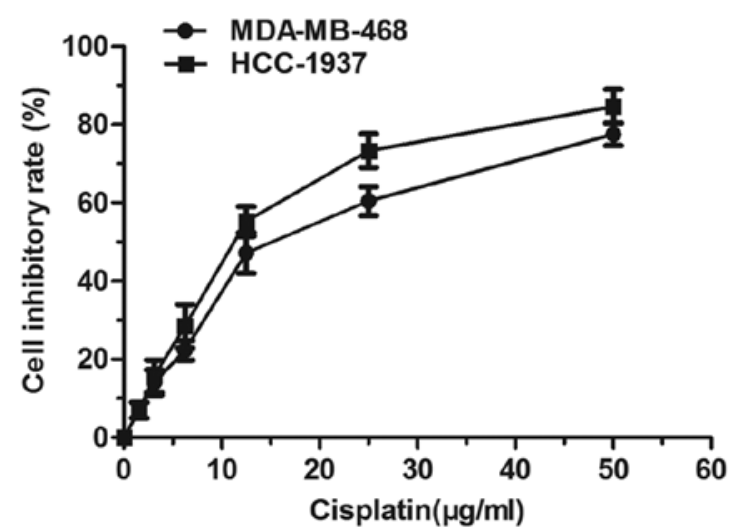

Figure 1. Inhibitory effects of cisplatin on MDA-MB-468 and HCC-1937 cell growth as determined by MTT viability assay. The cells were treated with 0 , $1.57,3.12,6.25,12.5,25$ and $50 \mu \mathrm{g} / \mathrm{ml}$ cisplatin for $48 \mathrm{~h}$. Then MTT assay was carried out to analyze the cell viability. Experiments were repeated three times in quadruplicates. Each data point represents the mean \pm SD.

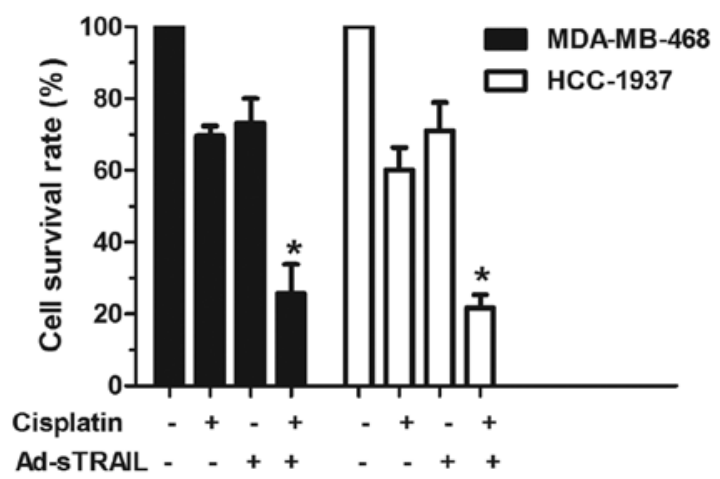

Figure 2. Inhibitory effects of combined treatment withAd-sTRAIL and cisplatin on MDA-MB-468 and HCC-1937 cell lines as determined by MTT viability assay. Cells were treated with cisplatin $(5 \mu \mathrm{g} / \mathrm{ml})$ and Ad-sTRAIL (MOI=10), alone or in combination for $48 \mathrm{~h}$. Cell viability was then analyzed by MTT method. Experiments were repeated three times in quadruplicates. Each data point represents the mean \pm SD (bars). Cisplatin plus Ad-sTRAIL treatment significantly inhibited the survival of MDA-MB-468 $\left({ }^{*} \mathrm{P}<0.05\right)$ and HCC-1937 ( $\mathrm{*}$ P<0.05) cells.

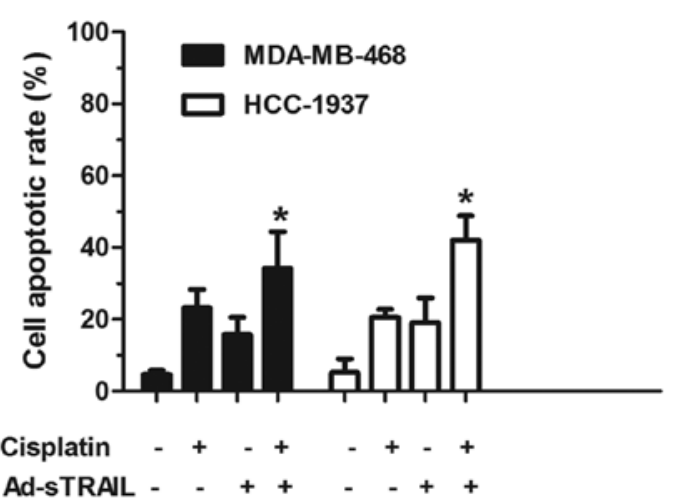

Figure 3. Detection of the apoptosis of MDA-MB-468 and HCC-1937 cells by flow cytometric assay. Cells were treated with cisplatin $(5 \mu \mathrm{g} / \mathrm{ml})$ and Ad-sTRAIL (MOI=10), alone or in combination for $48 \mathrm{~h}$. The cells were then harvested and stained with Annexin V-PE or PI to distinguish apoptotic and dead cells. All steps were conducted in accordance with the manufacturer's instructions. Finally, the stained cells were analyzed by flow cytometry. The percentage of apoptotic cells was calculated with FlowJo software. Each data point represents the mean $\pm \mathrm{SD}$ (bars). Cisplatin and Ad-sTRAIL treatment significantly induced the apoptosis of MDA-MB-468 ( $\mathrm{P}<0.05)$ and HCC$1937\left({ }^{*} \mathrm{P}<0.05\right)$ cells 


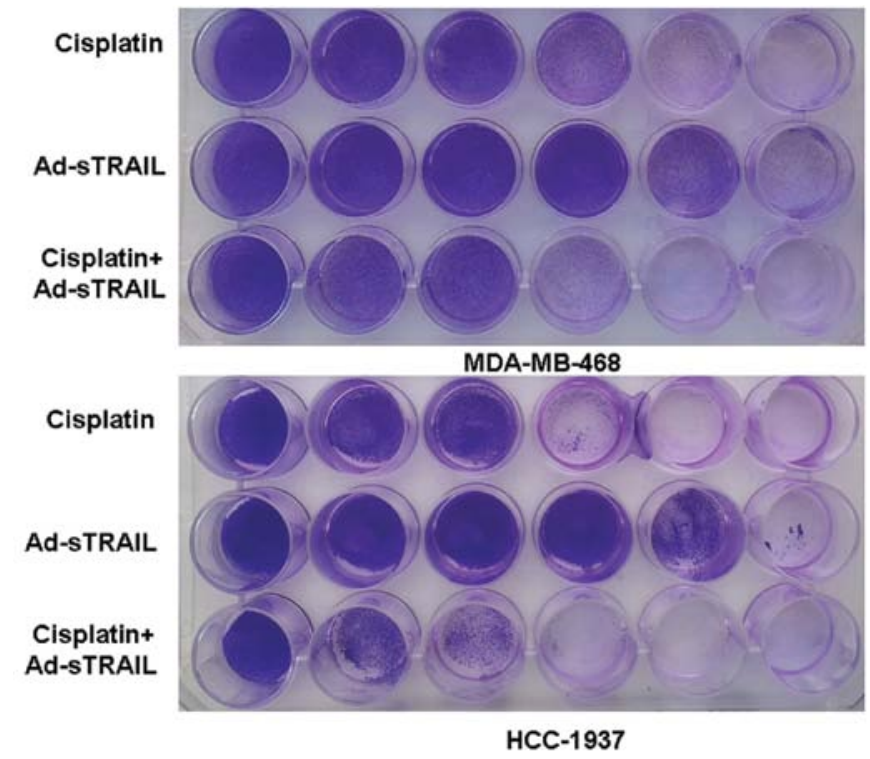

Figure 4. Enhanced suppressive effects of tumor cell proliferation by treatment with a combination of Ad-sTRAIL and cisplatin. From left to right, the concentration of cisplatin was $0,1.57,3.12,6.25,12.5$ and $25 \mu \mathrm{g} / \mathrm{ml}$. In the same order, the MOI of Ad-sTRAIL was $0,1,5,10,50$ and 100. Similarly, each well of the third row was treated with the same dosage of cisplatin and Ad-sTRAIL as the two wells above it. After 7 days of incubation, cells were stained with $2 \%$ crystal violet. The experiment was repeated three times with essentially the same results.

alone was dose-dependent (Fig. 4). Moreover, in the condition that the concentration of cisplatin was $6.25 \mu \mathrm{g} / \mathrm{ml}$ plus the MOI of Ad-sTRAIL was 10, the effect of the combination treatment was more effective than that of cisplatin $(25 \mu \mathrm{g} / \mathrm{ml})$ or Ad-sTRAIL (MOI=100) alone. The observations of the DAPI assay were consistent with the former results. Nuclear changes were noted which showed that few nuclei of apoptotic cells were noted in the control groups. In the groups receiving the combined treatment, large numbers of apoptotic cell nuclei were noted, which were more than in the groups treated with either agent alone (Fig. 5).
Ad-sTRAIL and cisplatin treatment downregulates the level of anti-apoptotic molecules cFLIP and BCL2L1. The effects of Ad-sTRAIL and cisplatin on the expression of anti-apoptotic molecules cFLIP and BCL2L1 in MDA-MB-468 and HCC-1937 cell lines were examined. The data in Fig. 6 indicate that, in the groups receiving either agent alone, the inhibition of cFLIP mRNA and BCL2L1 mRNA was more obvious than that in the control group. Moreover, the maximum effect of inhibition appeared in the group treated with both agents. In addition, TRAIL mRNA was significantly enhanced in groups treated with Ad-sTRAIL, while BCL2 mRNA did not show significant changes whether or not cells were treated with cisplatin.

Cisplatin upregulates DR5 and enhances the activation of caspase-8, thus enhancing the sensitivity to Ad-sTRAIL of breast cancer cells. We investigated the expression of DR5 in the 4 groups. Fig. 7 indicates that cisplatin enhanced the expression of DR5 in comparison to the control group. Expression of DR5 in the groups treated with Ad-sTRAIL was almost unchanged. Previous studies have confirmed that caspases play an important part in TRAIL-induced apoptosis (22). Fig. 7 shows that, in the groups treated with cisplatin, the activity of caspase- 8 was increased compared to that in the control groups. Furthermore, the groups treated with Ad-sTRAIL and cisplatin showed a maximum effect for the activity of caspase- 8 .

\section{Discussion}

Toxic side-effects and resistance of many tumors to currenttreatment strategies, particularly to chemotherapeutic strategies continue to be a main concern of cancer treatment. Thus, it is essential to develop more effective therapeutic modalities which are simultaneously specific for tumor cells with low toxicity for normal cells. The recombinant adenovirus, a nonpathogenic virus with a single-stranded DNA genome, has a broad host range and can be used to transduce breast cancer cells. Thus, it is presently being used in a number of clinical
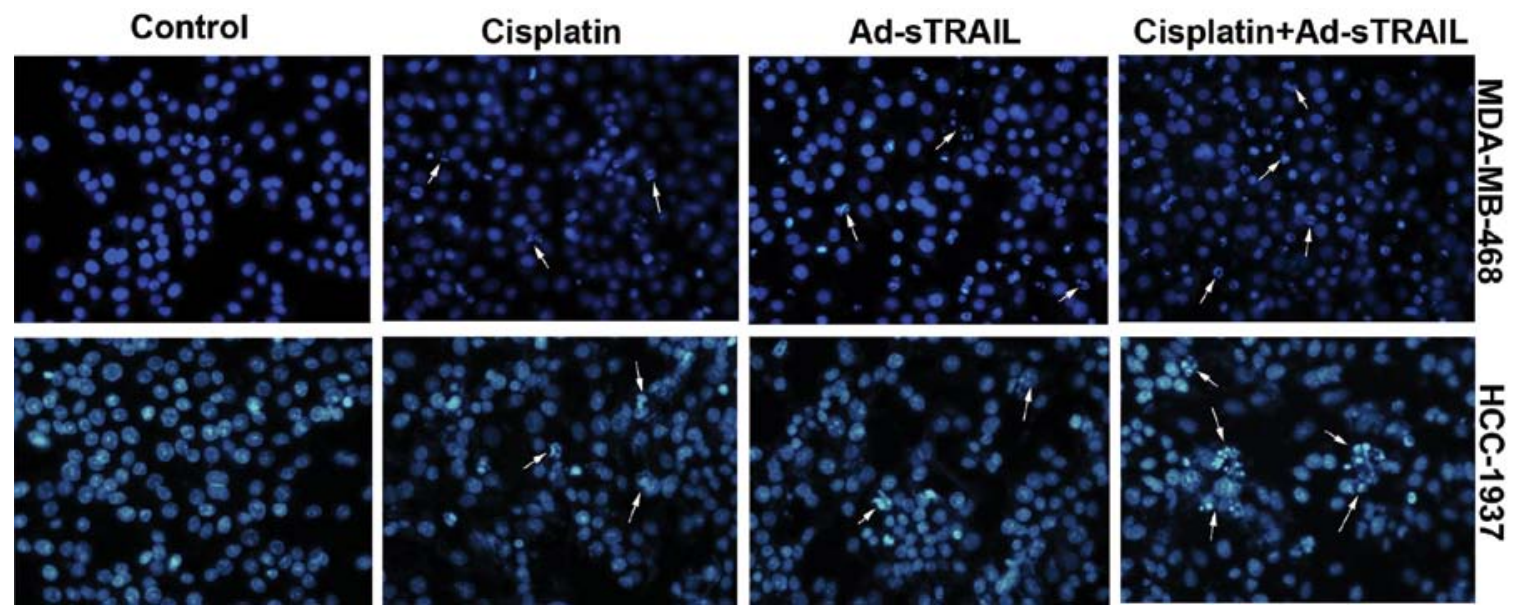

Figure 5. Analysis of cell morphology. MDA-MB-468 and HCC-1937 cells were treated with cisplatin $(5 \mu \mathrm{g} / \mathrm{ml}$ ) and Ad-sTRAIL (MOI=10), alone or in combination for $48 \mathrm{~h}$, and incubated with DAPI dye for $30 \mathrm{~min}$. Thereafter, chromatin condensation and nuclear fragmentation were observed under a fluorescence microscope. Normal cells were in different mitotic phases. (DAPI stain; original magnification, $\mathrm{x} 400$ ). 


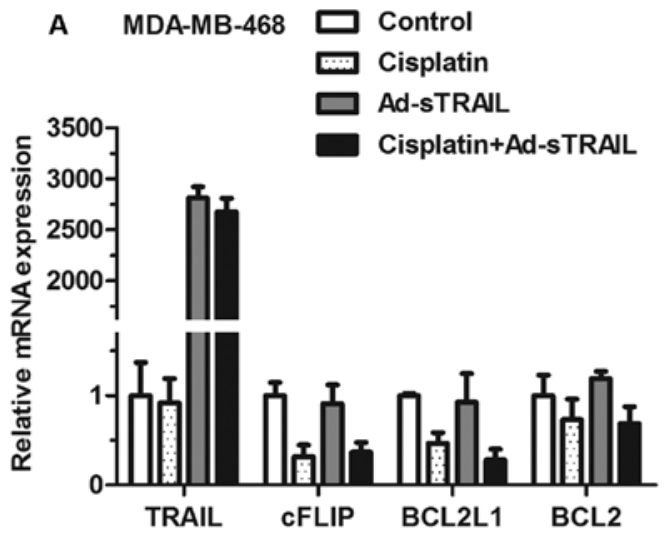

B

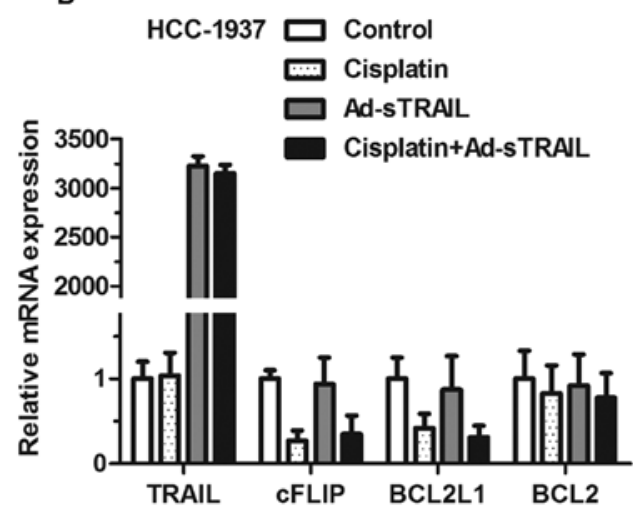

Figure 6. Quantitative PCR analysis of TRAIL, cFLIP, BCL2L1 and BCL2 mRNA expression levels in (A) MDA-MB-468 and (B) HCC-1937 cells Total mRNA was extracted from cells of the 4 groups and was detected by real-time fluorescence quantitative PCR analysis. GAPDH served as an internal control.

trials and may become a prospective component of antitumor therapy. Cisplatin is one of most efficacious chemotherapeutic drugs for breast cancer; however, it produces major toxicities to normal cells at the concentrations effective for cancer treatment. Therefore, the combination of chemotherapeutic agents and gene therapy is a potential efficacious strategy for breast cancer treatment (23).

TRAIL resistance is exhibited in many types of tumor cells; thus, attenuating its monotherapy in the clinic. Shin et al (24) reported that TRAIL-resistant human hepatocellular carcinoma becomes sensitive to TRAIL by co-treatment with cycloheximide and cisplatin, respectively. Furthermore, Jiang et al (25) also found that AAV-mediated TRAIL expression in conjunction with cisplatin demonstrated synergistic therapeutic effects on head and neck cancers. These studies demonstrated that the combined use of TRAIL and chemotherapeutic agents is promising. Thus, we applied Ad-sTRAIL and cisplatin concomitantly to two types of breast cancer cells. In the present study, we confirmed that MDA-MB-468 and HCC-1937 cells treated with Ad-sTRAIL and cisplatin exhibited significantly increased apoptosis than untreated cells. Moreover, the present study also confirmed that cisplatin promotes the sensitivity of cancer cells to TRAIL-induced apoptosis by the increased expression of DR5, suppression of cFLIP and BCL2L1 and activation of caspase-8.

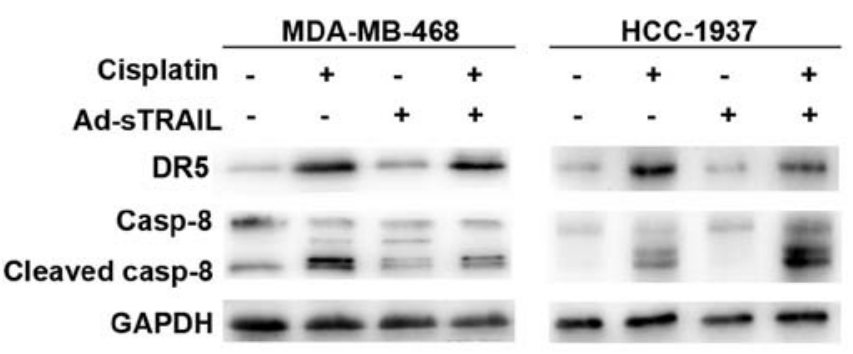

Figure 7. Identification of DR5 and caspase-8 (casp-8) by western blotting MDA-MB-468 and HCC-1937 cells were treated with cisplatin, Ad-sTRAIL or cisplatin plus TRAIL. Whole cell extracts were harvested and analyzed by western blotting. Equal protein loading was compared with that of GAPDH.

TRAIL induces apoptosis through binding to cell surface receptors. To date, five receptors of TRAIL have been identified. Among these, TRAIL-R1 (DR4), TRAIL-R2 (DR5), TRAIL-R3 (DcR1) and TRAIL-R4 (DcR2) are membranebound receptors. DR4 and DR5 contain a cytoplasmic death domain, while DcR1 and DcR2 are decoy receptors without a cytoplasmic death domain (26). TRAIL is shown to bind with DR4 and DR5, leading to the formation of Fas-associated protein with death domain (FADD). FADD and caspase-8 are recruited to form the death-inducing signaling complex (DISC). Activation of procaspase- 8 at the DISC triggers the downstream signaling cascades mainly through mitochondrial-dependent and mitochondrial-independent pathways. In the former pathway, activated caspase- 8 cleaves Bid thus forming truncated Bid (tBid) which translocates to the mitochondria causing release of cytochrome $c$, the loss of mitochondrial membrane potential and activation of caspase- 9 and -3 . In the latter pathway, activated caspase- 8 activates caspase- 3 directly. On the whole, activated caspase- 3 cleaves target substrates and finally leads to cell apoptosis in both pathways (27). Osteoprotegerin, a soluble receptor for TRAIL may act as a third decoy receptor (28).

Most tumor cells exhibit high expression of DR4 and DR5, yet with less or no DcR1 and DcR2. Therefore, TRAIL could promote the apoptosis of tumor cells by combining with DR4 and DR5. But in most normal tissues and cells, the expression of DcR1 and DcR2 dominates, and they bind to TRAIL thus protecting normal tissues from TRAIL-induced apoptosis (29). In the present study, cispatin treatment upregulated the expression of DR5 and thereafter sensitized breast cancer cells to TRAIL-induced apoptsis. The results indicate that the resistance of breast cancer cells to TRAIL may be partly due to the low expression of DR5.

The BCL2 family consisting of anti-apoptotic and proapoptotic members regulates cell apoptosis by inhibiting or promoting mitochondrial apoptosis signals. The former includes BCL2, BCL2L1 and Mcl-1, and the latter includes Bax, Bak, Bid and Bim $(30,31)$. The anti-apoptotic members of the BCL2 family carry out their actions by inhibiting the release of cytochrome $c$ from the mitochondria to the cytoplasm and by maintaining the integrity of the mitochondrial membrane by preventing mitochondrial swelling and membrane hyperpolarization $(32,33)$. In the present study, the expression of BCL2L1 mRNA was attenuated in cells following treatment of 
cisplatin, while BCL2 mRNA did not show significant changes whether or not cells were treated with cisplatin. Kim et al (34) performed functional genetic screening to isolate genes interfering with TRAIL-induced apoptosis using a cDNA retroviral library, and concluded that BCL2L1 but not BCL2 suppressed TRAIL-induced apoptosis in tumor cells. The present study further indicates that BCL2L1 may play an important role in the resistance of breast cancer cells to TRAIL.

cFLIP is a newly discovered apoptosis-inhibited protein which is composed of two isoforms: $\mathrm{cFLIP}_{\mathrm{s}}$ (a short form) and cFLIP $_{\mathrm{L}}$ (a long form). Both of them contain two death effector domains (DED) that bind to FADD, thus preventing caspase- 8 binding to FADD and inhibiting cell apoptosis mediated by TRAIL $(35,36)$. Wang et al $(37)$ reported that knockdown of cFLIP by a short hairpin RNA (shRNA) rendered resistant pancreatic cells sensitive to TRAIL-induced apoptosis through the cleavage of caspase- 8 and activation of the mitochondrial pathway. Brooks and Sayers (38) also demonstrated that treatment of human renal carcinoma cells with small interfering oligoribonucleotides (siRNA) for cFLIP caused a reduction in cFLIP protein and sensitized cells to TRAIL-mediated apoptosis. In the present study, we showed that the expression of cFLIP was significantly decreased in cells treated with cispaltin, and cells exposed to cisplatin plus TRAIL exhibited significantly enhanced apoptosis in comparison with the untreated cells. Therefore, the resistance of breast cancer cells to TRAIL was probably in part due to the overexpression of cFLIP proteins.

Based on the above-mentioned results, it was shown that TRAIL in combination with cisplatin showed excellent antitumor efficacy in MDA-MB-468 and HCC-1937 breast cancer cell lines by the increased expression of DR5, and decreased expression of cFLIP and BCL2L1. Simultaneously, the combination of cisplatin and Ad-sTRAIL attenuated the potential toxicity of cisplatin. These findings prove that the combination of cispaltin and Ad-sTRAIL is a potential therapeutic mode for breast cancer; and gene therapy may find its own niche in the comprehensive treatment of malignancies. Moreover, we demonstrated that the resistance of breast cancer to TRAIL may be due to the low expression of death receptors and overexpression of cFLIP; consequently, the mechanisms of the resistance of various types of tumors to TRAIL remain to be further clarified.

\section{Acknowledgements}

The present study was supported by grants from the Natural Science Foundation of the Shandong Province of China (Y2008C48), the Department of Education of the Shandong Province of China (J11LF05) and the Research Program of the Qingdao South District Municipal Science and Technology Commission (2011-5-004-YY).

\section{References}

1. Curado MP: Breast cancer in the world: incidence and mortality. Salud Publica Mex 53: 372-384, 2011.

2. Kamangar F, Dores GM and Anderson WF: Patterns of cancer incidence, mortality, and prevalence across five continents: defining priorities to reduce cancer disparities in different geographic regions of the world. J Clin Oncol 24: 2137-2150, 2006.
3. Kelley SK and Ashkenazi A: Targeting death receptors in cancer with Apo2L/TRAIL. Curr Opin Pharmacol 4: 333-339, 2004.

4. Ashkenazi A, Pai RC, Fong S, Leung S, Lawrence DA, Marsters SA, Blackie C, Chang L, McMurtrey AE, Hebert A, DeForge L, Koumenis IL, Lewis D, Harris L, Bussiere J, Koeppen H, Shahrokh Z and Schwall RH: Safety and antitumor activity of recombinant soluble Apo2 ligand. J Clin Invest 104: 155-162, 1999.

5. Ryo K, Kamogawa Y, Ikeda I, Yamauchi K, Yonehara S, Nagata S and Hayashi N: Significance of Fas antigen-mediated apoptosis in human fulminant hepatic failure. Am J Gastroenterol 95: 2047-2055, 2000.

6. Yan S, Qu X, Xu C, Zhu Z, Zhang L, Xu L, Song N, Teng Y and Liu Y: Down-regulation of Cbl-b by bufalin results in up-regulation of $\mathrm{DR}_{4} / \mathrm{DR}_{5}$ and sensitization of TRAIL-induced apoptosis in breast cancer cells. J Cancer Res Clin Oncol 138: 1279-1289, 2012.

7. Eggert A, Grotzer MA, Zuzak TJ, Wiewrodt BR, Ho R, Ikegaki N and Brodeur GM: Resistance to tumor necrosis factor-related apoptosis-inducing ligand (TRAIL)-induced apoptosis in neuroblastoma cells correlates with a loss of caspase-8 expression. Cancer Res 61: 1314-1319, 2001.

8. Hyer ML, Croxton R, Krajewska M, Krajewski S, Kress CL, Lu M, Suh N, Sporn MB, Cryns VL, Zapata JM and Reed JC: Synthetic triterpenoids cooperate with tumor necrosis factorrelated apoptosis-inducing ligand to induce apoptosis of breast cancer cells. Cancer Res 65: 4799-4808, 2005.

9. Zhang L and Fang B: Mechanisms of resistance to TRAILinducing apoptosis in cancer. Cancer Gene Ther 12: 228-237, 2005.

10. Singh TR, Shankar S, Chen X, Asim M and Srivastava RK: Synergistic interactions of chemotherapeutic drugs and tumor necrosis factor-related apoptosis-inducing ligand/Apo-2 ligand on apoptosis and on regression of breast carcinoma in vivo. Cancer Res 63: 5390-5400, 2003.

11. Hesry V, Piquet-Pellorce C, Travert M, Donaghy L, Jégou B, Patard JJ and Guillaudeux T: Sensitvity of prostate cells to TRAIL-induced apoptosis increases with tumor progression: DR5 and caspase 8 are key players. Prostate 66: 987-995, 2006.

12. Siddik ZH: Cisplatin: mode of cytotoxic action and molecular basis of resistance. Oncogene 22: 7265-7279, 2003.

13. Nagane M, Pan G, Weddle JJ, Dixit VM, Cavenee WK and Huang HJ: Increased death receptor 5 expression by chemotherapeutic agents in human gliomas causes synergistic cytotoxicity with tumor necrosis factor-related apoptosis inducing ligand in vitro and in vivo. Cancer Res 60: 847-853, 2000.

14. Boulikas T and Vougiouka M: Recent clinical trials using cisplatin, carboplatin and their combination chemotherapy drugs. Oncol Rep 11: 559-595, 2004.

15. Burtness B, Goldwasser MA, Flood W, Mattar B and Forastiere AA; Eastern Cooperative Oncology Group: Phase III randomized trial of cisplatin plus placebo compared with cisplatin plus cetuximab in metastatic/recurrent head and neck cancer: an Eastern Cooperative Oncology Group study. J Clin Oncol 23: 8646-8654, 2005.

16. Wiezorek J, Holland P and Graves J: Death receptor agonists as a targeted therapy for cancer. J Clin Cancer Res 16: 1701-1708, 2010.

17. Mandel RJ, Manfredsson FP, Foust KD, Rising A, Reimsnider S, Nash K and Burger C: Recombinant adeno-associated viral vectors as therapeutic agents to treat neurological disorders. Mol Ther 13: 463-483, 2006.

18. Wagner JA, Reynolds T, Moran ML, Moss RB, Wine JJ, Flotte TR and Gardner P: Efficient and persistent gene transfer of AAV-CFTR in maxillary sinus. Lancet 351: 1702-1703, 1998.

19. Wang Y, Huang F, Cai H, Wu Y, He G and Tan W: The efficacy of combination therapy using adeno-associated virus-TRAIL targeting to telomerase activity and cisplatin in a mice model of hepatocellular carcinoma. J Clin Cancer Res 136: 1827-1837, 2010.

20. Kanazawa T, Urabe M, Mizukami H, Okada T, Kume A, Nishino H, Monahan J, Kitamura K, Ichimura K and Ozawa K: $\gamma$-rays enhance rAAV-mediated transgene expression and cytocidal effect of AAV-HSVtk/ganciclovir on cancer cells. Cancer Gene Ther 8: 99-106, 2001. 
21. Livak KJ and Schmittgen TD: Analysis of relative gene expression data using real-time quantitative PCR and the 2(-Delta Delta C (T)) method. Methods 25: 402-408, 2001.

22. Lin J, Zhang Z, Zeng S, Zhou S, Liu BF, Liu Q, Yang J and Luo Q: TRAIL-induced apoptosis proceeding from caspase3-dependent and independent pathways in distinct HeLa cells. Biochem Biophys Res Commun 346: 1136-1141, 2006.

23. Shankar S and Srivastava RK: Enhancement of therapeutic potential of TRAIL by cancer chemotherapy and irradiation: mechanisms and clinical implications. Drug Resist Updat 7 139-156, 2004.

24. Shin EC, Seong YR, Kim CH, Kim H, Ahn YS, Kim K, Kim SJ, Hong SS and Park JH: Human hepatocellular carcinoma cells resist to TRAIL-induced apoptosis, and the resistance is abolished by cisplatin. Exp Mol Med 34: 114-122, 2002

25. Jiang M, Liu Z, Xiang Y, Ma H, Liu S, Liu Y and Zheng D: Synergistic antitumor effect of AAV-mediated TRAIL expression combined with cisplatin on head and neck squamous cell carcinoma. BMC Cancer 11: 54-65, 2011.

26. Liping X, Shuping Y and Kaladhar BR: Enhanced anticancer effect of the combination of cisplatin and TRAIL in triplenegative breast tumor cells. Mol Cancer Ther 10: 550-557, 2011.

27. Seol DW, Li J, Seol MH, Park SY, Talanian RV and Billiar TR Signaling events triggered by tumor necrosis factor-related apoptosis-inducing ligand (TRAIL): caspase- 8 is required for TRAIL-induced apoptosis. Cancer Res 61: 1138-1143, 2001.

28. Brunetti G, Colucci S, Rizzi R, Mori G, Colaianni G, Oranger A, Zallone A, Liso V and Grano M: The role of OPG/TRA IL complex in multiple meloma: the OPG/TRAIL complex in an in vitro osteoclastogenesis model derived from human multiple meloma-bone disease. Ann NY Acad Sci 1068: 334-340, 2006

29. Sheridan JP, Marsters SA, Pitti RM, Gurney A, Skubatch M, Baldwin D, Ramakrishnan L, Gray CL, Baker K, Wood WI, Goddard AD, Godowski P and Ashkenazi A: Control of TRAILinduced apoptosis by a family of signaling and decoy receptors. Science 277: 818-821, 1997.
30. Fulda S, Meyer E and Debatin KM: Inhibition of TRAIL-induced apoptosis by BCL2 overexpression. Oncogene 21: 2283-2294, 2002.

31. Van Geelen CM, de Vries EG and de Jong S: Lessons from TRAIL-resistance mechanisms in colorectal cancer cells: paving the road to patient-tailored therapy. Drug Resist Updat 7: 345-358, 2004

32. Vander Heiden MG, Chandel NS, Williamson EK, Schumacker PT and Thompson CB: Bcl-Xl regulates the membrane potential and volume homeostasis of mitochondria. Cell 91: 627-637, 1997.

33. Harris MH and Thompson CB: The role of the BCL2 family in the regulation of outer mitochondrial membrane permeability. Cell Death Differ 7: 1182-1191, 2000.

34. Kim IK, Jung YK, Noh DY, Song YS, Choi $\mathrm{CH}$, Oh BH, Masuda ES and Jung YK: Functional screening of genes suppressing TRAIL-induced apoptosis: distinct inhibitory activities of Bcl- $X_{L}$ and Bcl-2. Br J Cancer 88: 910-917, 2003

35. Lee TJ, Lee JT, Park JW and Kwon TK: Acquired TRAIL resistance in human breast cancer cells are caused by the sustained cFLIP(L) and XIAP protein levels and ERK activation. Biochem Biophys Res Commun 351: 1024-1030, 2006.

36. Bellail AC, Tse MC, Song JH, Phuphanich S, Olson JJ, Sun SY and Hao C: DR5-mediated DISC controls caspase- 8 cleavage and initiation of apoptosis in human glioblastomas. J Cell Mol Med 14: 1303-1317, 2010.

37. Wang P, Zhang J, Anita B, Jiang W, Judith H, Norman MK and Hao C: Inhibition of RIP and c-FLIP enhances TRAIL-induced apoptosis in pancreatic cancer cells. Cell Signal 19: 2237-2246, 2007.

38. Brooks AD and Sayers TJ: Reduction of the antiapoptotic protein cFLIP enhances the susceptibility of human renal cancer cells to TRAIL apoptosis. Cancer Immunol Immunother 54: 499-505, 2005. 